\title{
Editorial
}

\section{Progress in research on cystic fibrosis}

The Ninth International Cystic Fibrosis Congress was held in Brighton in June. These meetings take place every four years and provide an opportunity to assess progress in research on one of the commonest inherited disorders in the world. Over 1000 delegates attended the conference, of whom half were doctors or scientists and half were other health professionals, such as physiotherapists, dieticians, and social workers. The wide range of different professional groups attending, the number of different countries represented, and the presence of a sizable group of young adults with cystic fibrosis all combined to make the congress unique among medical meetings and undoubtedly contributed to its success.

Over 300 different contributions ${ }^{1}$ were presented and the wide variety of the topics covered make a comprehensive review impossible. For these reasons this editorial will concentrate only on a few areas of special interest and promise. The major subjects which I will ignore include the characteristics of the cystic fibrosis cell, studies of exocrine function and mucus, gastrointestinal pathophysiology, psychosocial aspects of cystic fibrosis, and the microbiology of the respiratory tract. These are all highly important and much valuable work was presented but it was difficult to see any major advance or new direction. In contrast, the fields of clinical management, ${ }^{2}$ prenatal diagnosis, carrier detection, ${ }^{3}$ and especially membrane transport ${ }^{4}$ all contained exciting new material and these will be discussed in some detail. The search for the basic defect by means of gene probe analysis is proceeding steadily and about $20 \%$ of the human genome has now been excluded with reasonable confidence. This branch of research is particularly tantalising as a DNA linkage may be found quite unpredictably at any moment by one of the many groups working their way through the genome chromosome by chromosome. Inevitably luck plays a part in this work and whoever stumbles on the correct sequence of genes first will be richly rewarded. This sense of imminent breakthrough ran through the whole meeting, and many workers are now convinced that very fundamental advances will be made before the next international congress in Australia in 1988.

Address for reprint requests: Dr Duncan Geddes, London Chest Hospital, London E2 9JX.

\section{Clinical management}

The three most important themes were new antibiotics against Pseudomonas aeruginosa, the move towards home treatment, and the role of supplemental nutrition.

Until there is better understanding of the underlying defect in the lung the clinical problem remains the treatment of persistent airway colonisation with Pseudomonas aeruginosa. No drug is able to sterilise the lung for long periods and so intermittent courses of intravenous antibiotics are the mainstay of treatment. Ceftazidime is a useful new agent with very powerful antipseudomonal activity. Although drug resistance may become a problem after repeated courses the early clinical trials have given encouraging results. No disadvantage has yet been demonstrated from using the drug as a single agent and side effects have been few. Also interesting is the new antibiotic ciprofloxacin. This is another highly active agent, with the advantage that it can be taken by mouth. Oral treatment would be a valuable advance as it would avoid many hospital admissions. Early experience with this drug is interesting and further studies are eagerly awaited. During the discussions about new antibiotics it became apparent that the current methods of assessment are inadequate. It is not enough to show that a new drug works; future studies should also look at the time to the next relapse and the frequency of further exacerbations. Similarly, there is still no agreement about the optimum length of a course of antibiotics, or the size or frequency of dose.

Several different centres reported their practice of home intravenous antibiotic treatment. Patients are taught to give their own injections during a brief stay in hospital to initiate treatment and establish optimum dosage. Subsequently the complete course of treatment can be given at home if the clinical condition permits. Some patients have learned to insert their own cannulas. Ceftazidime is particularly suitable for home treatment as is can be given alone as a low volume bolus three times daily. This form of treatment is still controversial and many fear that it may lead to falling standards of care. The advantages of continued work or education, however, together with added personal independence, are difficult to ignore. Furthermore, treatment may be given earlier in the course of an exacerbation, before symptoms are severe enough to require admission, and lung damage may actually be prevented. 
Supplemental nutrition also remains controversial but some trends were obvious. Long term home parenteral nutrition is not really suitable for these patients and is in any case probably unnecessary. The finding that short term intravenous feeding was followed by long term clinical improvement and weight gain ${ }^{5}$ draws attention to the importance of apathy and undereating in the malnutrition of cystic fibrosis. Anorexia combined with an increased nutritional requirement as a result of infection often results in simple dietary deficiency, and this may be more important than pancreatic insufficiency and malabsorbtion. It seems that if nutrition can be improved for a short period then the patient feels better and the appetite improves sufficiently for an adequate diet to be taken by mouth. For the few rẹmaining patients with severe malabsorbtion enteral supplementation by nocturnal nasogastric feeding may have a place, but airway irritability and the frequency of nasal polyps in cystic fibrosis make this approach difficult. A fine permanent jejunostomy tube may eventually be the answer but more experience is needed.

\section{Prenatal diagnosis and carrier detection}

After many false starts there is now real hope for accurate prenatal diagnosis. Brock ${ }^{6}$ has shown that alkaline phosphatase activity in amniotic fluid is much higher when the fetus has inherited cystic fibrosis than in the pregnancies of normal mothers and of carrier mothers when the fetus is unaffected. Although the numbers studied are still small and the discrimination is not perfect, the test is the best reported so far. The rise in the alkaline phosphase activity is associated with high levels of some other enzymes formed by microvillous membranes, and these are probably derived from fetal intestine. The test may therefore be a reflection of fetal gastrointestinal function. If so, some affected fetuses will inevitably be missed as the pancreas is variably defective. Furthermore, the enzyme levels change appreciably during the second trimester and so the results of amniocentesis performed before 18 weeks may be misleading. For these reasons the test must still be regarded as experimental and at risk pregnancies should be notified whenever possible to a research centre such as Dr DJH Brock's in Edinburgh. In this way it may be possible to work out the true value of the method at the same time as providing some guarded information to the parents. When the genetic defect is eventually known an accurate first trimester diagnosis should be possible by analysing DNA obtained from a biopsy specimen of chorionic villi. This approach must wait for the success of gene probe analysis.
Isoelectric focusing of serum has revealed the presence of a "cystic fibrosis protein." This is found in homozygotes and in intermediate amounts in heterozygotes but not in the normal population (allowance has to be made for a carrier rate in the normal population of about 1:25). This protein was first identified by Wilson ${ }^{7}$ in 1975 and his findings have since been confirmed in several laboratories. The presence of such a protein indicates that a heterozygote test is feasible. The protein is, however, present in very small amounts and its identification is far too difficult for any population screening to be contemplated. Research workers have therefore concentrated on raising a monoclonal antibody to the cystic fibrosis protein to simplify the analysis. After many years of frustration there are indications that progress is being made. As yet no definitive results have been reported, but a few smiles were visible on the right faces and cautious optimism is in the air.

\section{Membrane transport of fluid and ions}

Membrane transport of fluid and ions is probably the most exciting and rapidly moving field in research on cystic fibrosis today for three main reasons. In the first place, the sweat gland and the airway epithelium have now been shown to share a common defect; secondly, the defect suggests likely mechanisms for the pathogenesis of cystic fibrosis lung disease; and, finally, the abnormalities may be modified by drugs and this raises the hope of treatment to prevent or delay lung damage.

\section{THE SWEAT GLAND}

Since the 1950s high concentrations of sodium and chloride ions in the sweat have been recognised as the single most reliable abnormality in cystic fibrosis. The cause of this change has, however, only recently been worked out. In the normal person the primary secretion contains $\mathrm{Na}^{+}, \mathrm{Cl}^{-}$, and water and the ionic concentrations are the same as those in plasma. As this primary secretion passes along the duct $\mathrm{Na}^{+}, \mathrm{Cl}^{-}$, and water are reabsorbed to produce a hypotonic solution on the skin. In cystic fibrosis the primary secretion is normal and so the abnormality must lie in the control of reabsorption in the duct. In a series of brilliantly conceived and executed experiments Quinton and coworkers have demonstrated that the abnormality is almost certainly due to an impairment of $\mathrm{Cl}^{-}$permeability of the membrane of the ductal epithelium. In the first series of experiments ${ }^{8}$ the intraductal electrical potential was studied in isolated perfused sweat glands obtained from the skin of normal individuals and patients with cystic fibrosis. The glands were 


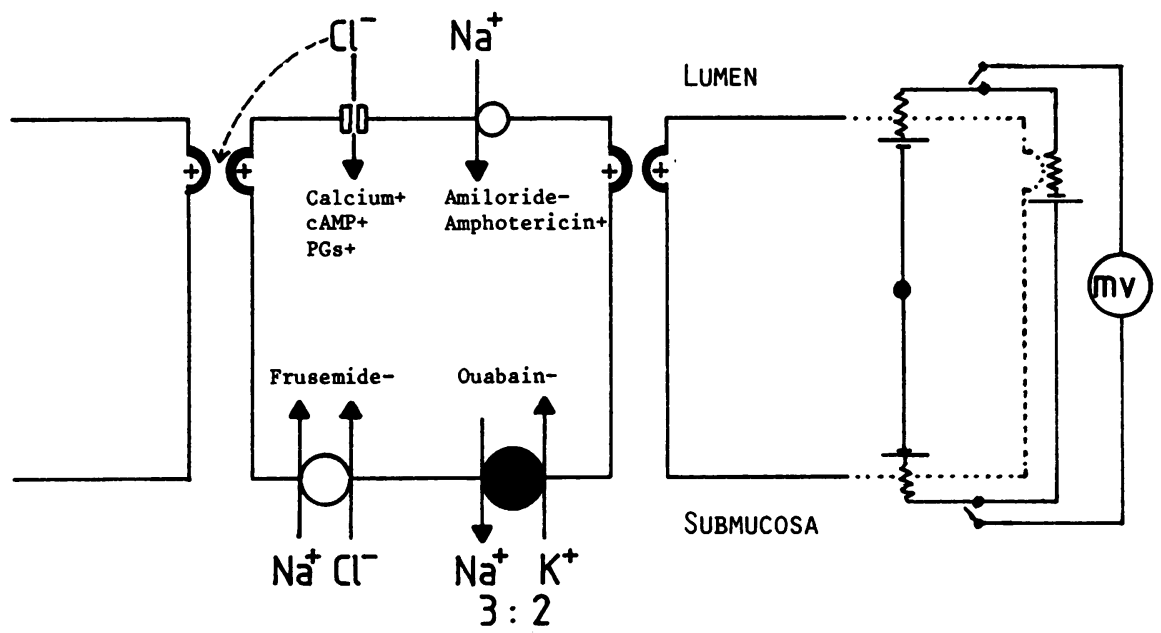

Ion transport in airway epithelium. $\mathrm{Cl},-, \mathrm{Na}^{+}, \mathrm{K}^{+}-$negative chloride and positive sodium and potassium ions; $c A M P \longrightarrow$ cyclic adenosine monophosphate; PGs-prostaglandins.

perfused with solutions of different ionic compositions. The most important findings were that the duct in cystic fibrosis has a much more negative intraluminal potential difference than normal, suggesting an excess of $\mathrm{Cl}^{-}$ions (too little $\mathrm{Cl}^{-}$reabsorption) or a lack of $\mathrm{Na}^{+}$ions (too much $\mathrm{Na}^{+}$reabsorption). Substitution of $\mathrm{Cl}^{-}$by sulphate in the perfusate (sulphate cannot cross cell membranes) converted the potential difference of normal ducts into the cystic fibrosis range and so suggested that $\mathrm{Cl}^{-}$ impermeability was the likely defect. Further studies, in which active $\mathrm{Na}^{+}$transport was blocked with ouabain, gave results consistent with this interpretation. In a second series of experiments ${ }^{9}$ individual sweat ducts were studied in vivo and measurements of intraluminal potential difference and rates of secretion of $\mathrm{Na}^{+}, \mathrm{Cl}^{-}$, and water were made. The increased negative potential difference was confirmed and the actual rates of ductal reabsorption of $\mathrm{Na}^{+}$and $\mathrm{Cl}^{-}$were calculated. Reabsorption of both ions was less than normal and transport of $\mathrm{Cl}^{-}$was reduced more than $\mathrm{Na}^{+}$. There are two important conclusions from these studies-firstly, that the intraluminal potential difference is abnormally negative in the sweat duct in cystic fibrosis and, secondly, that this is due to $\mathrm{Cl}^{-}$impermeability.

\section{AIRWAY ION TRANSPORT (see figure)}

The observation that most closely mirrors the abnormality of the sweat gland is the finding of an increased negative intraluminal potential difference in the airway. Knowles et al in $1981^{10}$ reported that the inferior nasal turbinate in cystic fibrosis had a potential difference of $-60 \mathrm{mv}$, compared with $-25 \mathrm{mv}$ in normal controls. The same was found in the intrapulmonary airways, although only a few patients have been studied and the differences were less striking. The altered nasal potential difference has been confirmed by other workers. When amiloride is applied to the luminal surface (amiloride blocks luminal $\mathrm{Na}^{+}$transport) the potential difference falls more in cystic fibrosis than in normal individuals. This led the authors to speculate that excessive $\mathrm{Na}^{+}$transport was the cause of the increased negative potential difference in cystic fibrosis. The hypothesis of enhanced $\mathrm{Na}^{+}$transport out of the lumen is attractive as water would be expected to follow, resulting in dehydration of airway secretions and compromised mucociliary clearance.

Further work on airway ion transport has used three approaches: firstly, in vivo studies on the nasal epithelium or, with the use of a bronchoscope, the intrapulmonary airways; secondly, in vitro studies on excised nasal polyps; and, finally, studies on airway epithelia maintained in explants or tissue culture.

\section{Airway in vivo}

Studies of the airway in vivo are somewhat limited in scope since the range of possible measurements is small. By measuring the potential difference in the nose with and without amiloride while the ionic constituents of the superfusate were changed Knowles et $a l^{11}$ concluded that $\mathrm{Cl}^{-}$impermeability was the main defect. Bronchoscopic studies attempting to measure the salt and water content of the airway fluid have been inconclusive but the approach is nevertheless important as direct measurements in vivo are few and it is difficult to see how else the 
water content of respiratory secretions can be estimated. Furthermore, ion transport probably varies with airway generation ${ }^{12}$ and these changes can be studied during bronchoscopy. Potential difference in normal individuals is less negative in the lobar bronchi than the trachea, but there are no data from the, peripheral airways in cystic fibrosis and this is probably the most relevant site for investigation. Because the clinical indications for bronchoscopy in cystic fibrosis are few progress is slow. In this context it is particularly interesting that the transplanted lung in a recipient with cystic fibrosis had a normal intraluminal potential difference.

\section{Nasal polyps in vitro}

The data from these studies are confusing and difficult to interpret. Boucher and coworkers ${ }^{12}$ have shown that the short circuit current generated by polyp epithelium is an accurate reflection of $\mathrm{Na}^{+}$ transport and that this current is greater in polyps from patients with cystic fibrosis than atopic controls. They interpreted this as indicating increased $\mathrm{Na}^{+}$transport by the apical membrane. In contrast they also found evidence of $\mathrm{Cl}^{-}$impermeability. It is not yet clear whether disordered transport of one ion affects the transport of the other. The matter is made more difficult by the responses to different agonists and antagonists. The amiloride induced reduction in transepithelial conductance is somewhat less in cystic fibrosis than in normal individuals, which suggests that the number of actively transporting $\mathrm{Na}^{+}$channels is not increased. Beta agonists, in contrast, increase $\mathrm{Na}^{+}$transport in cystic fibrosis but not normal polyps, suggesting a qualitative difference. $\mathrm{Cl}^{-}$secretion is stimulated by $\beta$ agonists, protaglandins, and calcium ionophore in normal epithelia but not in cystic fibrosis tissue. At present therefore the evidence may point to a more generalised defect in ion transport than $\mathrm{Cl}^{-}$impermeability but its nature is far from clear.

\section{Explants and tissue culture}

The need for more detailed studies of transport mechanisms and their control is obvious and so good in vitro test systems are essential. Two techniques are now being developed: one requires the growing of human bronchial explants in nude mice and this is proceeding well; the second approach is to maintain a monolayer of human tracheal cells in tissue culture and a normal transepithelial potential difference across the monolayer has been demonstrated. Although this progress is encouraging the problem of regional differences in airway transport may be difficult to overcome. It is not clear whether the in vitro systems will reliably reflect these differences. Neither is it clear whether the differences them- selves are due to variation in local cell populations or to controlling mechanisms that might not be represented outside the intact organ.

\section{Conclusions}

THE PRIMARY ABNORMALITY

Any attempt to produce a simple unified hypothesis for the epithelial abnormality in cystic fibrosis is premature and cannot explain all the diverse findings. Two different sets of observations need to be reconciled. In the first place, there is evidence of $\mathrm{Cl}^{-}$impermeability alone in the sweat duct, while both $\mathrm{Cl}^{-}$and $\mathrm{Na}^{+}$transport may be altered in the airway. Secondly, the transport defect does not appear to affect all tissues. $\mathrm{Cl}^{-}$secretion by the gastric mucosa is not grossly impaired in cystic fibrosis, and while there is some evidence of altered renal handling of $\mathrm{Na}^{+14}$ this is certainly not a major problem. Nevertheless, two general possibilities exist.

The first is concerned with chloride impermeability, which could be due to a mutation in a single membrane protein. The other cellular changes might then be due to the altered electrochemical environment or to a spillover effect of secondary changes in intracellular mediators. Alternatively, a general defect of control of ion pathways might be expressed differently in different epithelia according to their specialised function.

\section{TREATMENT}

The science of airway ion transport is relatively young. Nevertheless, some agents have been shown to affect the different transport sites specifically (fig). This raises the possibility of modifying the transport defect with drugs. The problems with this line of thought are, firstly, that the pathways are shared by cells throughout the body, so that a desirable change in the airways might produce an undesirable change elsewhere; and, secondly, that the concentrations of drugs given in pharmacological experiments are unacceptably high for clinical use. Both of these problems are common to other branches of clinical pharmacology and both can be minimised by topical application of the drug. Nebulisation is a possible approach in the airways and very preliminary trials of high doses of inhaled amiloride are under way. It will be a long time before any genuinely corrective treatment for the airways is available but there is now real hope that the lung damage of cystic fibrosis may be in part preventable.

DUNCAN GEDDES
Brompton and London Chest Hospitals
London
London 


\section{References}

${ }^{1}$ Lawson D, ed. Cystic fibrosis: horizons. Chichester: John Wiley and Sons, 1984.

${ }^{2}$ Lawson D, ed. Cystic fibrosis: horizons. Chichester: John Wiley and Sons, 1984:84-138.

${ }^{3}$ Lawson D, ed. Cystic fibrosis: horizons. Chichester: John Wiley and Sons, 1984:1-28.

${ }^{4}$ Lawson D, ed. Cystic fibrosis: horizons. Chichester: John Wiley and Sons, 1984:167-28.

${ }^{5}$ Sheperd R, Cooksey WGF, Cooke WD. Improved growth and clinical, nutritional and respiratory changes in response to nutritional therapy in cystic fibrosis. Pediatr 1980; 7:351-7.

${ }^{6}$ Brock DJH. Amniotic fluid alkaline phosphatase isoenzymes in early prenatal diagnosis of cystic fibrosis. Lancet 1983;ii:941-3.

7 Wilson GB, Fudenberg HH, Jahn TL. Studies on cystic fibrosis using isoelectric focussing: an assay for detection of cystic fibrosis homozygote and heterozygote carriers in serum. Pediatric Res 1975;9:635-40.
${ }^{8}$ Quinton PM. Chloride impermeability in cystic fibrosis. Nature 1983;301:421-2.

${ }^{9}$ Quinton PM, Bijman J. Higher bioelectric potentials due to decreased chloride absorption in sweat glands of patients with cystic fibrosis. $N$ Engl J Med 1983;308:1189-91.

${ }^{10}$ Knowles M, Gatzy J, Boucher R. Increased bioelectrical potential difference across respiratory epithelia in cystic fibrosis. N Engl J Med 1981;305:1489-95.

" Knowles M, Gatzy J, Boucher R. Relative ion permeability of normal and cystic fibrosis nasal epithelium. $J$ Clin Invest 1983;71:1410-7.

${ }^{12}$ Boucher R, Bromberg PA, Gatzy J. Airway transepithelial potential in vivo: species and regional differences. $J$ Appl Physiol 1980;48:169-76.

${ }^{13}$ Knowles M, Stutts M, Spock A, Fischer J, Gatzy J, Boucher RC. Science 1983;221:1067-70.

${ }^{14}$ Berg U, Kusoffsky E, Strandvik B. Renal function in cystic fibrosis with special reference to renal sodium handling. Acta Paed Scand 1982;71:833-8. 